\title{
Analysis of triplet repeats in the huntingtin gene in Japanese families affected with Huntington's disease
}

Naoki Masuda, Jun Goto, Nobuhiro Murayama, Masahiko Watanabe, Ikuko Kondo, Ichiro Kanazawa

that the allele of seven repeats could be critical in Huntington's disease.

We here report the results of analysis of the CAG and CCG repeat polymorphism in Japanese Huntington's disease families and a normal population. mal controls was performed. The size the CAG repeat ranged from 37 to 95 repeats in affected subjects and from seven to 29 in normal controls. A significant correlation was found between the age of onset and the CAG expansion. The length of the expanded repeat is unstable in meiotic transmission and large increases occur in paternal transmission. At the same time the CCG repeat polymorphism adjacent to the CAG repeat was analysed and haplotypes of HD chromosomes were identified. Strong linkage disequilibrium was found between the CAG repeat expansion and an allele of (CCG) $)_{10}$ in Japanese HD chromosomes. It is distinct from that described previously in western populations. Western HD chromosomes strongly associate with an allele of (CCG) $)_{7}$. Possible mechanisms underlying the disequilibrium in Japan are discussed.

( $\mathcal{F}$ Med Genet 1995;32:701-705)

Department of

Clinical Neurology and

Neuroscience,

Institute for Brain

Research,

Faculty of Medicine,

University of Tokyo,

7-3-1 Hongo,

Bunkyo-ku,

Tokyo 113, Japan

N Masuda

J Goto

M Watanabe

I Kanazawa

Department of Hygiene,

Faculty of Medicine,

Ehime University,

Ehime, Japan

N Murayama

I Kondo

School of

Pharmaceutical

Sciences,

Showa University,

Tokyo, Japan

N Murayama

Correspondence to: Dr Goto.

Received 31 January 1995 Revised version accepted for publication 28 April 1995
Huntington's disease (HD) is a progressive neurodegenerative disorder which is clinically characterised by chorea, cognitive decline, and emotional disturbance; it is inherited in an autosomal dominant manner. An unstable and abnormally expanded CAG repeat in the huntingtin (IT15) gene has been shown to cause the disease by the Huntington's Disease Collaborative Research Group. ${ }^{1}$ The prevalence of $\mathrm{HD}$ is approximately 3 to 8 per 100000 in most western populations ${ }^{23}$; however, the frequency is less than one-tenth in Japan. ${ }^{45} \mathrm{We}$ have carried out linkage analysis of Japanese $\mathrm{HD}$ families ${ }^{67}$ and found that the markers flanking the HD gene, D4S127, D4S95 and D4S43, are closely linked in Japanese families affected with the disease.

There is another triplet sequence, a CCG repeat, immediately $3^{\prime}$ adjacent to the CAG repeat in the huntingtin gene. The triplet sequence is also polymorphic, ${ }^{8}$ alleles of seven or 10 repeats are predominant in populations, and strong linkage disequilibrium between the former allele and HD has been shown in white populations. ${ }^{9-11}$ As one hypothesis which could explain this observation it has been proposed

\section{Materials and methods}

FAMILY STUDIES

The HD families analysed had been examined by neurologists or psychiatrists or both. Information on clinical status, sex, age at onset, and pedigree structure was recorded for all $\mathrm{HD}$ families. Ascertainment of a patient was based on characteristic clinical features and family history. The age of onset was defined as the age at which choreic movements or psychiatric impairment were first observed.

\section{DNA ISOLATION}

Genomic DNA was isolated from either lymphoblastoid cell lines or the buffy coat layer of fresh blood by standard extraction methods. $^{12}$

\section{PCR ANALYSIS}

PCR amplification of the triplet repeat sequence in the huntingtin gene was performed using the primers $\mathrm{HD}-1, \mathrm{HD}-2, \mathrm{HD}-3$, and $\mathrm{HD}-4 \mathrm{~F}$, which have been described previously. ${ }^{1813}$ The primers HD-1 (ATGAAGGCCTTCGAGTCCCTCAAGTCCTTC) and HD-2 (AAACTCACGGTCGGTGCAGCGGCTCCTCAG) produce a product containing the $\mathrm{CAG}$ repeat and the GC rich region, which includes the polymorphic CCG repeat. The primers HD-3 (GGCGGTGGCGGCTGTTGCTGCTGCTGCTGC) and HD-4F (GCAGCAGCAGCACCAAAGCCGCCACCGCC) are complementary and are at the boundary between the CAG repeat and the CCG repeat; the primers $\mathrm{HD}-1$ and $\mathrm{HD}-3$ amplify only the CAG repeat sequence, and the primers HD-2 and HD-4F amplify the GC rich region. These three PCR amplifications were performed for all DNA samples analysed. The amplified alleles were separated on a $5 \%$ polyacrylamide gel containing $7 \mathrm{~mol} / \mathrm{l}$ urea. Allele sizes were determined by comparison with the sizes of the amplified products of pHE5A and $\mathrm{pHE} 5 \mathrm{Na}$ and $\mathrm{M} 13 \mathrm{mp} 18$ sequence ladders. pHE5A and pHE5Na are the plasmids constructed as follows. Genomic DNA from a 

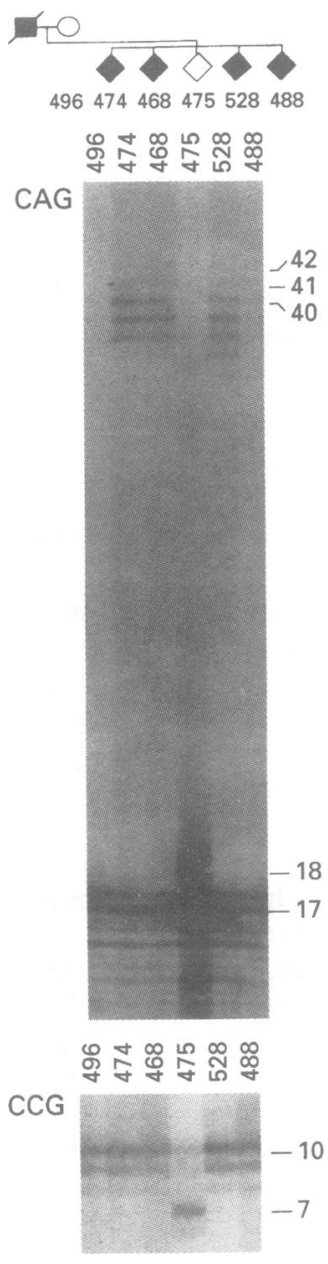

HAPLOTYPING

The haplotype of each chromosome studied was determined by comparison of the lengths of products of the three sets of PCR amplification, and was deduced and confirmed by phase determination where two or three generations were available for molecular analysis.

\section{Results}

DISTRIBUTION OF CAG REPEAT NUMBER

Our linkage studies showed that Japanese HD families were tightly linked to chromosome $4 \mathrm{p} 16.3 .^{67}$ All $20 \mathrm{HD}$ families previously studied were analysed for determination of number of the CAG repeat. A typical autoradiograph of

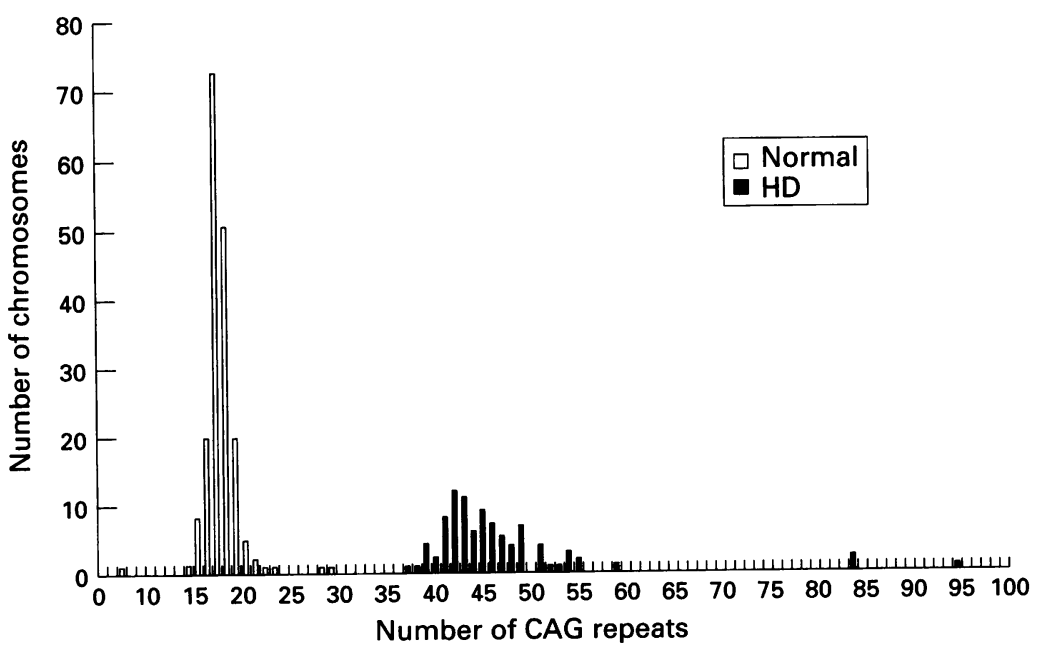

Figure 2 Distribution of the CAG repeat in the huntingtin gene in fapanese $H D$ and normal populations. Open bar =normal chromosomes. Filled bar $=H D$ chromosomes. one of the families is shown in fig 1 . It was confirmed that all affected subjects with HD show expansion of the CAG repeat (data not shown).

Additional Japanese HD families that were not studied for linkage analysis were also analysed. Molecular data were obtained from 92 affected subjects from 58 unrelated HD families. A control group consisted of 185 unrelated non-HD chromosomes which were identified in the families studied. The distribution of normal and abnormal CAG repeat number is shown in fig 2 . The normal range varied from seven to 29 repeats (mode 17 repeats) and the observed heterozygosity and PIC in the normal Japanese population are 0.742 and 0.706 , respectively. The HD range is from 37 to 95 and the mode is 42 . The peaks of the normal and HD ranges are well separated and the two distributions do not overlap.

AGE OF ONSET AND CAG REPEAT LENGTH

Fig 3 shows the relationship between age of onset and the CAG repeat size in both alleles in affected subjects. A significant negative correlation $(n=77, r=-0.709, p<0.001)$ was evident for the relationship between the age of onset and the number of longer CAG repeats. The regression curve was derived according to the formula (CAG repeat number) $=$ $112-17 \cdot 72 \times \ln$ (age of onset).

Data on age of onset were obtained from 22 father-child pairs and five mother-child pairs in this cohort. The Wilcoxon signed rank test showed that children with paternal transmission developed symptoms significantly earlier than their fathers $(p=0.0001)$, whereas differences between mother-child pairs were not significant $(p=0 \cdot 124)$. The mean difference between father-child pairs was $20 \cdot 23$ (SE $2 \cdot 69)$ years, and $12 \cdot 20(\mathrm{SE} 6 \cdot 29)$ years between mother-child pairs. Molecular data of CAG expansion were obtained from 20 father-child pairs and 14 mother-child pairs. The difference in CAG repeat size on transmission of the HD chromosome was calculated and the results are shown in fig 4 . On paternal transmission the repeat size increased by 4.05 (SE 1.98 ) repeats on average, whereas the size decreased by 0.36 (SE 0.86 ) repeats on maternal transmission. The Wilcoxon signed rank test showed that children with paternal transmission had significantly larger expansions than their father $(p=0.015)$, whereas expansion from affected mothers to their children was not significant $(p=0.685)$. This study contained three children with the rigid form of HD and in all cases their HD mutations were inherited from their father.

\section{CCG REPEAT POLYMORPHISM AND}

HUNTINGTON'S DISEASE

The CCG repeat sequence immediately $3^{\prime}$ adjacent to the CAG repeat is polymorphic in white populations. ${ }^{8-11}$ The frequencies of the CCG repeat alleles in the normal Japanese population is shown in the table. Alleles of seven and 10 repeats occur in the majority of 


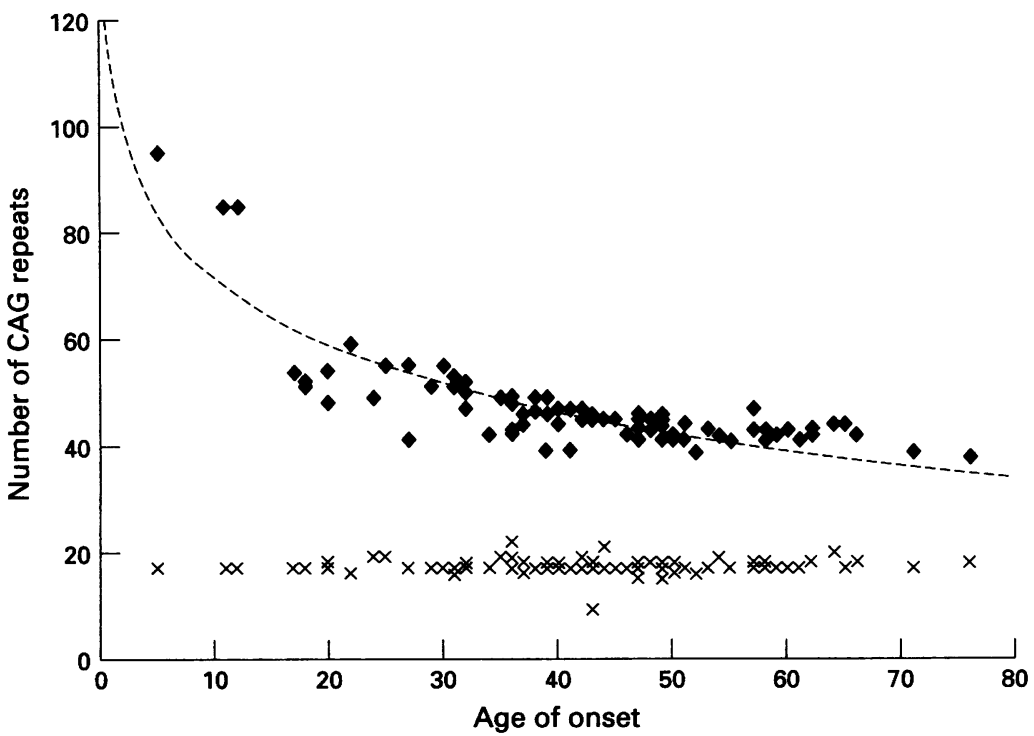

Figure 3 Relationship between the number of $C A G$ repeats and the age of onset. Diamonds = expanded allele, crosses = normal allele. The broken line is the regression curve which is of the formula (CAG repeat number) $=112-17.72 \times \ln$ (age of onset).

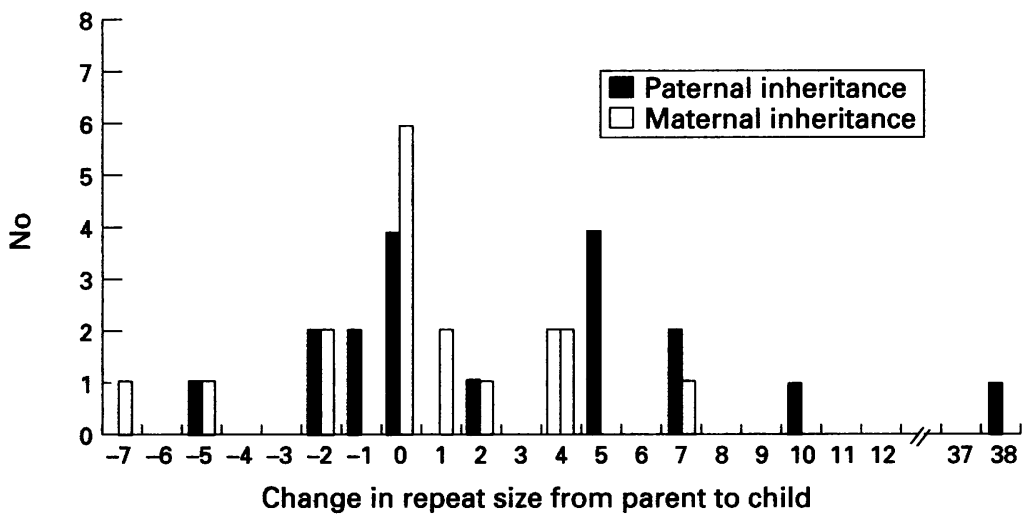

Figure 4 Variation of CAG repeat size on transmission of Huntington's disease chromosomes.

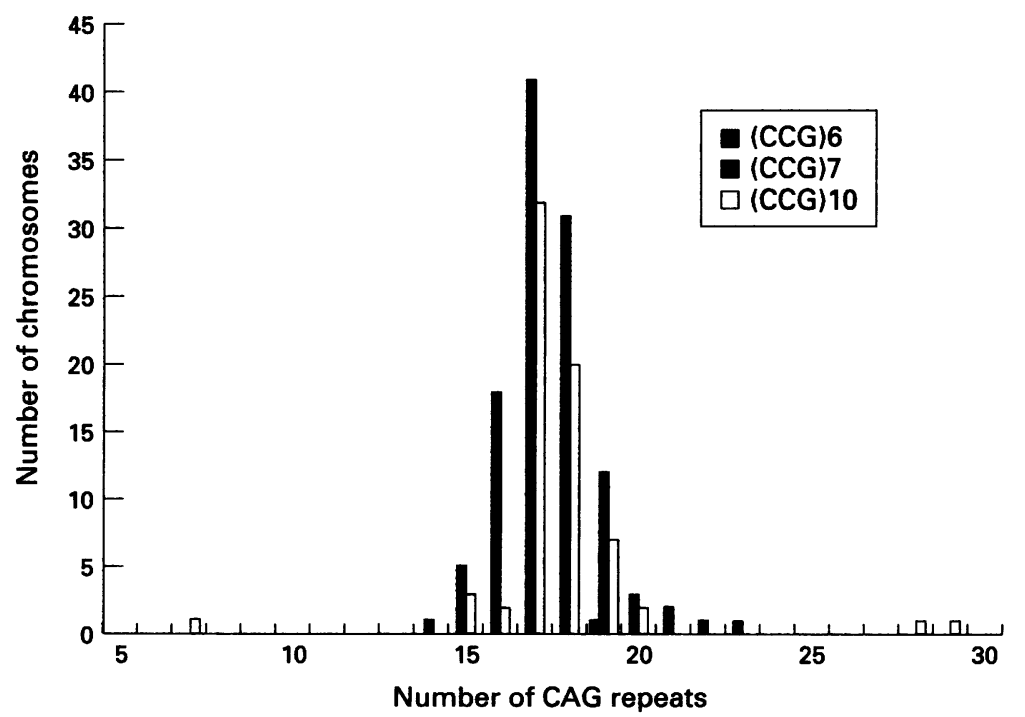

Figure 5 Haplotype of the CAG and CCG repeat polymorphism in the normal fapanese population. Hatched bar $=(C C G)_{6}$ allele. Filled bar $=(C C G)_{7}$ allele. Open bar= $(C C G)_{10}$ allele.

the Japanese population. The heterozygosity and PIC for the CCG repeat polymorphism in the Japanese population is 0.483 and 0.372 , respectively.
Allele frequencies of the CCG repeat polymorphism in the fapanese population

\begin{tabular}{lrrrrr}
\hline (CCG)n & \multicolumn{2}{c}{ Control } & & \multicolumn{2}{l}{$H D$} \\
\cline { 2 - 3 } \cline { 6 - 6 } \cline { 5 - 6 } & \multicolumn{1}{c}{ No } & $\%$ & & No & \multicolumn{1}{c}{$\%$} \\
\hline 6 & 1 & 0.5 & & 0 & 0.0 \\
7 & 115 & $62 \cdot 2$ & & 9 & 15.5 \\
8 & 0 & 0.0 & & 0 & 0.0 \\
9 & 0 & 0.0 & & 0 & 0.0 \\
10 & 69 & 37.3 & & 49 & 84.5 \\
Total & 185 & & & 58 & \\
\hline
\end{tabular}

$\chi^{2}=39 \cdot 39(p=0 \cdot 0001)$.

Recent studies have shown that more than $90 \%$ of $\mathrm{HD}$ chromosomes of western descent have the seven repeat allele and strong linkage disequilibrium between this allele and HD is evident. ${ }^{9-11}$ We first analysed the 20 families which had been previously used for linkage analysis. In only three families, the seven repeat allele cosegregated with the HD mutation; however, the 10 repeat allele cosegregated in 17 families. Fig 1 shows a family in which the 10 repeat allele unambiguously cosegregates with the expansion of the CAG repeat. Additional Japanese HD chromosomes were analysed and haplotypes were identified for a total of 58 unrelated Japanese HD chromosomes (table). Forty-nine HD chromosomes $(85.5 \%)$ cosegregated with the 10 repeat allele, whereas only nine $(15.5 \%)$ carried the seven repeat allele. The observed allele frequencies for the CCG repeat polymorphism in the HD population are significantly different from those obtained from the normal population $\left(\chi^{2}=39.39\right.$, $\mathrm{df}=2, \mathrm{p}=0.0001)$. This result supports strong linkage disequilibrium between $\mathrm{HD}$ and the CCG allele with 10 repeats in the Japanese population.

HAPLOTYPE ANALYSIS OF THE CAG AND CCG REPEAT POLYMORPHISM IN THE NORMAL JAPANESE POPULATION

Fig 5 shows the distributions of CAG repeat length of normal chromosomes which have a different CCG repeat allele. The mean CAG repeat size is 17.45 (SE 0.13 ) for $(\mathrm{CCG})_{7}$ chromosomes and 17.65 (SE 0.13) for (CCG) chromosomes. The latter is slightly larger than the former, although the difference is not significantly statistically ( $t$ value $=0.7, p=0.4839$ ) and the two distributions are indistinguishable $\left(\chi^{2}=15 \cdot 78, \mathrm{df}=12, \mathrm{p}=0 \cdot 2013\right)$.

The primers HD-1 and -2 , which have been previously described, ${ }^{1}$ amplify the sequence containing both CAG and CCG repeats and the products show combined polymorphism. Heterozygosity and PIC are calculated as $\mathbf{0 . 8 4 6}$ and 0.829 , respectively, and the distribution of alleles have small twin peaks (data not shown), which result from the two predominant alleles of the CCG repeat.

\section{Discussion}

EXPANSION OF THE CAG REPEAT

Analysis of the CAG repeat showed that the range of expansion of the repeat was between 37 and 95 (mean $=42$ ), that of normal chromosomes was between seven and 29 , and alleles 
containing repeats ranging from 30 to 36 were not found. The distribution of the expanded repeat of HD was similar to that for North American and European populations described previously. ${ }^{14-22}$ It is difficult to define precisely the smallest size of the CAG repeat expansion which causes the disorder. In most studies numbers around 37 were reported as the lowest numbers for HD of European descent: 35 for the Scottish population, ${ }^{20} 36$ for American, ${ }^{19}$ 37 for Italian, ${ }^{21} 38$ for Canadian, ${ }^{16} 39$ for German, ${ }^{17} 39$ for Danish, ${ }^{18}$ and 41 for Russian. ${ }^{22}$

A significant correlation was found for the relationship between the age of onset and the CAG repeat expansion. The longer the CAG repeat, the younger the age of onset. A similar relationship was described for the HD population of white descent. ${ }^{14-22}$ The inverse relationship is clear for juvenile onset $\mathrm{HD}$, but for adult onset cases any given number of repeats can be associated with an age of onset that varies by decades.

We obtained molecular data from $20 \mathrm{HD}$ father-child pairs and 14 mother-child pairs affected with HD. A significant increase of CAG repeat size on paternal transmission of $\mathrm{HD}$ and greater instability of paternally transmitted repeats has been shown in western HD families. ${ }^{1420}$ Analysis of Japanese affected parent-offspring pairs in this study also showed that on paternal transmission there appeared to be a younger age of onset together with the longer CAG repeat, but not on maternal transmission. Anticipation corresponds to expansion of the CAG repeat and it occurs in paternal transmission.

The above features of the CAG repeat are mostly similar to those of western populations.

\section{THE CCG REPEAT POLYMORPHISM}

The CCG repeat polymorphism is composed of the two predominant alleles (CCG) ${ }_{7}$ and $(\mathrm{CCG})_{10}$, and some other minor alleles in Japanese or western populations. Frequencies of both major alleles in the Japanese population were not significantly different from those in western populations which have been described previously..$^{9-11}$ Other minor alleles are rare in Japan, compared with data from western or black people, ${ }^{9-1122}$ and we identified only one chromosome with the $(\mathrm{CCG})_{6}$ allele in this study.

The prevalence of HD varies considerably among different ethnic groups. The frequency of the disease is very low in Japan and less than one-tenth of the prevalence in western countries. It was suggested that the low frequency of $\mathrm{HD}$ reflects the origins of the HD mutation. The original HD gene was considered to be western European in origin and subsequently was thought to have spread to Japan by emigration. ${ }^{2}$ Haplotype analysis of HD chromosomes provides a clue to the origins of the HD gene. It is interesting to note that there is a striking discrepancy between Japanese and western $\mathrm{HD}$, in terms of a CCG repeat allele showing strong association with the CAG repeat expansion. In fact, strong linkage disequilibrium is found between Japanese HD chromosomes and (CCG) $)_{10}$, whereas western HD chromosomes are strongly associated with (CCG) ${ }_{7}{ }^{9-11}$ The most plausible explanation for this discrepancy is that HD mutations in these two races originated from different ancestral lineages; the western HD mutation is mostly derived from a chromosome containing $(\mathrm{CCG})_{7}$ in western Europe, while Japanese HD is mostly from a (CCG) $)_{10}$ associated chromosome in the ancestors of the Japanese population. Although the mutation rate for $\mathrm{HD}$ was estimated to be extremely low, ${ }^{23}$ recent molecular genetic findings in HD suggest that new mutation events appear to occur more frequently than considered previously. ${ }^{23}$ Two groups described several new mutations for HD that were shown by molecular analysis of expansion of a CAG repeat. ${ }^{24}$ In addition, analysis of HD chromosomes with multiallelic markers was reported to show many different haplotypes, suggesting a variety of independent HD mutations. ${ }^{26}$ Therefore, in addition to the hypothesis of spread of the HD gene from western Europe as the origin of Japanese HD, it is reasonable to postulate that a new mutational event in the Japanese ancestral population might contribute to the present $\mathrm{HD}$ patients in Japan.

In addition to the founder effect, we can consider other hypotheses to account for the disequilibrium of the CCG repeat polymorphism. First, one could assume that a cisacting element links to the CCG repeat and predisposes to expansion of the CAG repeat. The CCG repeat sequence itself can be considered to have an effect on the CAG repeat expansion. Although it has been proposed that the $(\mathrm{CCG})_{7}$ allele could be critical in $\mathrm{HD},{ }^{10}$ Japanese HD chromosomes associate strongly with the $(\mathrm{CCG})_{10}$ allele. Therefore, it appears unlikely that a particular allele of the CCG repeat polymorphism might be involved in the mechanism of the disease. Another possibility is that relatively longer CAG repeat alleles in the normal range could be prone to mutate to expansion. The mean length of the CAG repeat of chromosomes containing $(C C G)_{10}$ was slightly longer than that of chromosomes carrying (CCG) ${ }_{7}$, although the difference was not significant. Longer alleles of the CAG repeat polymorphism, which constitute a very small fraction in our cohort, could be mostly associated with an allele of (CCG) $)_{10}$ in Japan and might constitute a reservoir providing premutations. Finally, one could speculate that the threshold of CAG repeat size to predispose to expansion may vary in a chromosome having a different sized allele of the CCG polymorphism. The threshold in a chromosome carrying (CCG) $)_{10}$ might be smaller than in a $(\mathrm{CCG})_{7}$ chromosome. However, the distributions of CAG repeat size appear to be similar for both $(\mathrm{CCG})_{10}$ and $(\mathrm{CCG})_{7}$ chromosomes (data not shown), though we have little molecular data about HD chromosomes carrying $(\mathrm{CCG})_{7}$.

We wish to thank the members of the HD families and their We wish to thank the members of the HD families and their Grant-in-Aid for Scientific Research on Priority Areas and Education, Science, and Culture, Japan. 
1 The Huntington's Disease Collaborative Research Group. A novel gene containing a trinucleotide repeat that is expanded and unstable on Huntington's disease chromosomes. Cell 1993;72:971-83.

2 Hayden MR. Huntington's chorea. New York: Springer-Verlag, 1981 .

3 Harper PS. Huntington's disease. London: Saunders, 1991

Kishimoto K, Nakamura M, Sotokawa Y. Population genetics study of Huntington's chorea in Japan. Annu Rep Res Inst Environ Med 1957:9:195-211.

5 Kanazawa I. On the prevalence rate of Huntington's disease in Ibaraki prefecture. In: Annual Report of the Research Committee of CNS Degenerative Diseases 1982. Tokyo: Ministry of Health and Welfare of Japan, 1983:151-6.

6 Kanazawa I, Kondo I, Ikeda J, et al. Studies on DNA markers (D4S10 and D4S43/S127) genetically linked to Huntington's disease in Japanese families. Hum Genet 1990;85:257-60.

7 Watanabe $M$, Kondo I, Nissato S, et al. A linkage study with DNA markers (D4S95, D4S115, and D4S111) in Japanese Huntington disease families. fpn $\mathcal{f}$ Hum Genet 1993;38:193-201.

8 Rubinsztein DC, Leggo J, Barton DE, Ferguson-Smith MA. Site of (CCG) polymorphism in the HD gene. Nature

9 Andrew SE, Goldberg YP, Theilmann J, et al. A CCG repeat polymorphism adjacent to the CAG repeat in the Huntington's disease gene: implications for diagnostic accuracy and predictive testing. Hum Mol Genet 1994;3: 65-7.

10 Barron LH, Rae A, Holloway S, et al. A single allele from the polymorphic CCG rich sequence immediately $3^{\prime}$ to the unstable CAG trinucleotide in the IT 15 cDNA shows almost complete disequilibrium with Huntington's disease chromosomes in the Scottish population. Hum Mol Genet 1994;3:173-5.

11 Novelletto A, Persichetti F, Sabbadini G, et al. Polymorphism analysis of the huntingtin gene in Italian families affected with Huntington's disease. Hum Mol Genet 1994; 3:1129-32.

12 Sambrook J, Fritsch EF, Maniatis T. Molecular cloning: a laboratory manual. 2nd ed. New York: Cold Spring Harbor Laboratory manual. 2nd ed.

13 Warner JP, Barron LH, Brock DJH. A new polymerase chain reaction (PCR) assay for the trinucleotide repeat that is unstable and expanded on Huntington's disease chromosomes. Mol Cell Probes 1993;7:235-9.
14 Duyao M, Ambrose C, Myers R, et al. Trinucleotide repeat length instability and age of onset in Huntington's disease. Nature Genet 1993;4:387-92.

15 Snell RG, MacMillan JC, Cheadle JP, et al. Relationship between trinucleotide repeat expansion and phenotype
variation in Huntington's disease. Nature Genet 1993;4: variation

16 Andrew SE, Goldberg YP, Kremer B, et al. The relationship between trinucleotide (CAG) repeat length and clinical features of Huntington's disease. Nature Genet 1993;4 398-403.

17 Zühlke C, Reiss O, Schröder K, et al. Expansion of the (CAG)n repeat causing Huntington's disease in 352 patients of German origin. Hum Mol Genet 1993;2: 1467-9.

18 Nørremølle A, Riess O, Epplen JT, et al. Trinucleotide repeat elongation in the Huntingtin gene in the Huntington's disease patients from 71 Danish families. Hum Mol Genet 1993;2:1475-6.

19 Stine OC, Pleasant N, Franz ML, et al. Correlation between the onset age of Huntington's disease and length of the trinucleotide repeat in IT-15. Hum Mol Genet 1993;2: 1547-9.

20 Barron LH, Warner JP, Porteous M, et al. A study of the Huntington's disease associated trinucleotide repeat in the Huntington's disease associated trinucleotide repeat

21 Novelletto A, Persichetti F, Sabbadini G, et al. Analysis of the trinucleotide repeat expansion in Italian families affected with Huntington's disease. Hum Mol Genet 1994; 3:93-8.

22 Illarioshkin $\mathrm{SN}$, Igarashi $\mathrm{S}$, Onodera $\mathrm{O}$, et al. Trinucleotide repeat length and rate of progression of Huntington's disease. Ann Neurol 1994;36:630-5.

23 Rubinsztein DC, Amos W, Leggo J, et al. Mutational bias provides a model for the evolution of Huntington's disease and predicts a general increase in disease prevalence. Nature Genet 1994;7:525-30.

24 Myers RH, MacDonald ME, Koroshetz WJ, et al. De novo expansion of a (CAG)n repeat in sporadic Huntington's disease. Nature Genet 1993;5:168-73.

25 Goldberg YP, Kremer B, Andrew SE, et al. Molecular analysis of new mutations for Huntington's disease: inanalysis of new mutations for Huntington's disease: intermediate alle

26 MacDonald ME, Novelletto A, Lin C, et al. The Huntington's disease candidate region exhibits many different haplotypes. Nature Genet 1992;1:99-103. 